\title{
EXPERIMENTAL REALIZATION OF BINARY SIGNALS TRANSMISSION BASED ON SYNCHRONIZED LORENZ CIRCUITS
}

\author{
C. Posadas-Castillo ${ }^{1}$, C. Cruz-Hernández² \& R. Núnez-Pérez² \\ ${ }^{1}$ Faculty of Enginnering Mechanic and Electrical (FIME), \\ Nuevo León Autonomous University (UANL), \\ Pedro de Alba s/n Cd. Universitaria San Nicolas de los Garza, Nuevo León, México. \\ cornelio_posadas@hotmail.com \\ ${ }^{2}$ Electronics and Telecommunications Department, \\ Scientific Research and Advanced Studies Center of Ensenada (CICESE), \\ Km. 107 Carretera Tijuana-Ensenada, Ensenada, B.C., 22860, México. \\ ccruz@cicese.mx
}

Received: May $24^{\text {th }}$, 2002. Accepted: November $18^{\text {th }}, 2002$

\section{ABSTRACT}

In this paper, an experimental study of practical realization to transmit private binary signals using chaos is presented. In particular, we use a Generalized Hamiltonian systems approach to synchronize two unidirectionally coupled Lorenz circuits with the receiver being given by an observer. Experimental results are in close accordance with the numerical simulations.

\section{RESUMEN}

Este trabajo presenta un estudio experimental relacionado con la comunicación de información privada, empleando caos, con base en la sincronía de dos circuitos de Lorenz por formas hamiltonianas y el diseño de un observador para el receptor. Los resultados experimentales coinciden con las simulaciones numéricas.

KEYWORDS: Synchronization, Chaos, Lorenz system, Passivity based observers, Private communication.

\section{INTRODUCTION}

Since Pecora and Carroll reported the discovery of synchronized chaos in [1], the possibility of secure communications using chaos has received considerable attention. Synchronization of chaotic systems has in recent years become an area of active research. Different approaches are proposed and being pursued (see, e.g., [1]-[16] and the references therein).

Data encryption using chaotic dynamics was reported in the early 1990s as a new approach for signal encoding that differs from the conventional methods that use numerical algorithms as the encryption key. Thereby, the synchronization of chaotic systems plays an important role in chaotic communications. In particular, several 
techniques, such as chaotic masking [17], chaotic switching [17]-[19] and chaotic parameter modulation [20] have been developed.

Our objective in this paper consists in describing an experimental study of practical realization to transmit private binary signals based on synchronized chaotic systems. In particular, we use a Generalized Hamiltonian systems and observer approach developed in [14] to synchronize two unidirectionally coupled Lorenz circuits. We enumerate several advantages over the existing methods:

- It enables synchronization be achieved in a systematic way and clarifies the issue of deciding on the nature of the output signal to be transmitted;

- it can be successfully applied to several well-known chaotic oscillators;

- it does not require the computation of any Lyapunov exponent;

- it does not require initial conditions belonging to the same basin of attraction.

This paper is organized as follows. In Section 2, we present the system description to transmit binary information by chaotic switching. In Section 3, we obtain the synchronization of two Lorenz systems through a Generalized Hamiltonian systems and observer approach. In Section 4, we present the stability analysis related to the synchronization process. In Section 5, we show the physical system implementation and experimental results: 5.1 synchronization and 5.2 binary communication. Finally, in Section 6, we give some concluding remarks.

\section{SYSTEM DESCRIPTION}

In this section, we describe the communication system based on synchronized chaos and its operating principles. Figure 1 shows a block diagram to transmit binary messages by chaotic switching. We will use a Lorenz system as chaos generator. The state equations are given by [21]

$$
\begin{gathered}
\dot{x}_{1}=\sigma\left(x_{2}-x_{1}\right), \\
\dot{x}_{2}=r x_{1}-x_{2}-x_{1} x_{3}, \\
\dot{x}_{3}=x_{1} x_{2}-b x_{3},
\end{gathered}
$$

with $\sigma=10, r=28$, and $b=8 / 3$ the Lorenz system exhibits chaotic behavior.

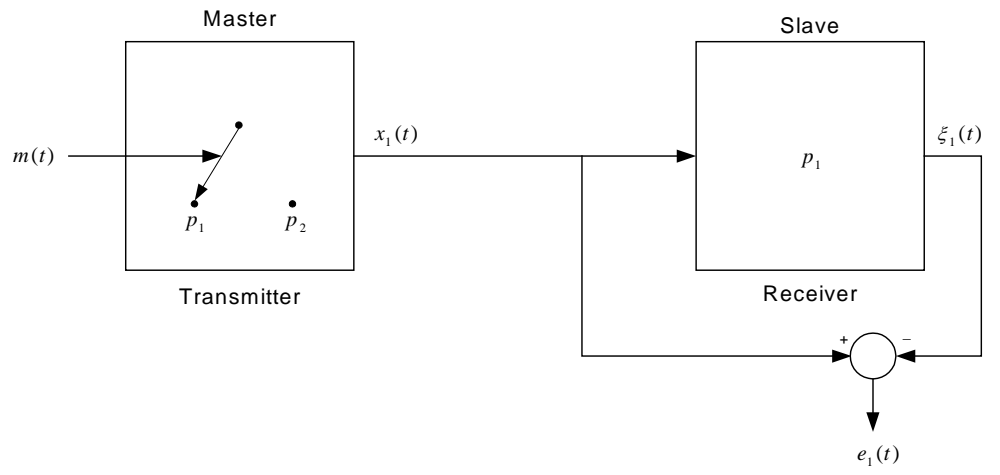

Figure 1. Block diagram to transmit private binary messages by chaotic switching

For private communication purposes here we apply the synchronized Lorenz circuits to chaotic switching. In this technique, the message $m(t)$ is supposed to be a binary message, and is used to modulate one or more parameters of the (switching) transmitter, i.e. $m(t)$ controls a switch whose action changes the parameter values of the transmitter. Thus, according to the value of $m(t)$ at any given time $t$, the transmitter has either the parameter set value $\mathrm{p}_{1}$ or the parameter set value $\mathrm{p}_{2}$. At the receiver, $m(t)$ is decoded by using the synchronization error 
$\left(e_{1}(t)=x_{1}(t)-\xi_{1}(t)\right)$ to decide whether the received message corresponds to one parameter value, or the other (it can be interpreted as an one or a zero). In the next section we describe the Generalized Hamiltonian systems approach to synchronize two unidirectionally coupled Lorenz circuits. Synchronization is thus between the transmitter and receiver dynamics with the receiver being given by an observer.

\section{SYNCHRONIZATION OF LORENZ SYSTEM}

Consider the following n-dimensional autonomous system

$$
\dot{x}=f(x), \quad x \in R^{n},
$$

which represents a circuit exhibiting a chaotic behavior. Following the approach provided in [14], many physical systems described by Eq. (2) can be written in the following "Generalized Hamiltonian" canonical form,

$$
\dot{x}=J(x) \frac{\partial H}{\partial x}+S(x) \frac{\partial H}{\partial x}+F(x)
$$

where $H(x)$ denotes a smooth energy function which is globally positive definite in $R^{n}$. The column gradient vector of $H$, denoted by $\partial H / \partial x$, is assumed to exist everywhere. We frequently use quadratic energy function $H(x)=1 / 2 x^{T} M x$ with $M$ being a, constant, symmetric positive definite matrix. In such case, $\partial H / \partial x=M x$. The square matrices, $J(x)$ and $S(x)$ satisfy, for all $x \in R^{n}$, the following properties, which clearly depict the managing structure of the system, $J(x)+J^{T}(x)=0$ and $S(x)=S^{T}(x)$. The vector field $J(x) \partial H / \partial x$ exhibits the conservative part of the system and it is also referred to as the workless part, or workless forces of the system; and $S(x)$ depicting the working or nonconservative part of the system. For certain systems, $S(x)$ is negative definite or semidefinite. In such cases, the vector field is addressed to as the dissipative part of the system. If, on the other hand, $S(x)$ is positive definite, positive semidefinite, or indefinite, it clearly represents, respectively, the global, semi-global and local destabilizing part of the system. In the last case, we can always (although nonuniquely) descompose such an indefinite symmetric matrix into the sum of a symmetric negative semidefinite matrix $R(x)$ and a symmetric positive semidefinite matrix $N(x)$. And $F(x)$ represents a locally destabilizing vector field.

We consider a special class of Generalized Hamiltonian systems with destabilizing vector field and linear output map, given by

$$
\begin{array}{ll}
\dot{x}=J(y) \frac{\partial H}{\partial x}+(I+S) \frac{\partial H}{\partial x}+F(y), & x \in R^{n}, \\
y=C \frac{\partial H}{\partial x}, & y \in R^{m}
\end{array}
$$

where $S$ is a constant symmetric matrix, not necessarily of definite sign. The matrix / is a constant skew symmetric matrix. The vector variable $y$ is referred to as the system output. The matrix $C$ is a constant matrix. 
We denote the estimate of the state vector $x$ by $\xi$, and consider the Hamiltonian energy function $H(\xi)$ to be the particularization of $H$ in terms of $\xi$. Similarly, we denote by $\eta$ the estimated output, computed in terms of the estimated state $\xi$. The gradient vector $\partial H(\xi) / \partial \xi$ is, naturally, of the form $M \xi$.

A dynamic nonlinear state observer for the system (4) is

$$
\begin{aligned}
& \dot{\xi}=J(y) \frac{\partial H}{\partial \xi}+(I+S) \frac{\partial H}{\partial \xi}+F(y)+K(y-\eta) \\
& \eta=C \frac{\partial H}{\partial \xi}
\end{aligned}
$$

where $K$ is a constant vector, known as the observer gain.

The state estimation error, defined as $e(t)=x(t)-\xi(t)$ and the output estimation error, defined as $e_{y}(t)=y(t)-\eta(t)$, are governed by

$$
\begin{gathered}
\dot{e}=J(y) \frac{\partial H}{\partial e}+(I+S-K C) \frac{\partial H}{\partial e}, \quad e \in R^{n}, \\
e_{y}=C \frac{\partial H}{\partial e}, \quad e_{y} \in R^{m},
\end{gathered}
$$

where the vector, $\partial H / \partial e$ actually stands, with some abuse of notation, for the gradient vector of the modified energy function, $\partial H(e) / \partial e=\partial H / \partial x-\partial H / \partial \xi=M(x-\xi)=M e$. Below, we set, when needed, $I+S=W$. We say that the receiver system (5) synchronizes with the transmitter system (4), if $e(t) \rightarrow 0$ as $t \rightarrow \infty$.

Lorenz system. To synchronize the Lorenz circuit using the Generalized Hamiltonian systems approach we use the Eq. (1). The set of state equations describing Lorenz system in Hamiltonian Canonical form with a destabilizing field (master) is given by

$$
\left[\begin{array}{c}
\dot{x}_{1} \\
\dot{x}_{2} \\
\dot{x}_{3}
\end{array}\right]=\left[\begin{array}{ccc}
0 & \frac{1}{2} \sigma & 0 \\
-\frac{1}{2} \sigma & 0 & -x_{1} \\
0 & x_{1} & 0
\end{array}\right] \frac{\partial H}{\partial x}+\left[\begin{array}{ccc}
-\sigma^{2} & \frac{1}{2} \sigma & 0 \\
\frac{1}{2} \sigma & -1 & 0 \\
0 & 0 & -b
\end{array}\right] \frac{\partial H}{\partial x}+\left[\begin{array}{c}
0 \\
r x_{1} \\
0
\end{array}\right]
$$

taking as the Hamiltonian energy function the scalar function

$$
H(x)=\frac{1}{2}\left[\frac{1}{\sigma} x_{1}^{2}+x_{2}^{2}+x_{3}^{2}\right]
$$

The destabilizing vector field evidently calls for $y=x_{1}=\left[\begin{array}{lll}\sigma & 0 & 0\end{array}\right] \partial H / \partial x$ to be used as the output of the master (7). The matrices $C$, S and / are given by 


$$
C=\left[\begin{array}{lll}
\sigma & 0 & 0
\end{array}\right], \quad S=\left[\begin{array}{ccc}
-\sigma^{2} & \frac{1}{2} \sigma & 0 \\
\frac{1}{2} \sigma & -1 & 0 \\
0 & 0 & -b
\end{array}\right], \quad I=\left[\begin{array}{ccc}
0 & \frac{1}{2} \sigma & 0 \\
-\frac{1}{2} \sigma & 0 & 0 \\
0 & 0 & 0
\end{array}\right]
$$

The pair of matrices $(C, S)$ already constitutes a pair of detectable, but nonobservable, matrices. Even though the addition of the matrix / to $S$ does not improve the lack of observability, the pair $(C, W)=(C, S+\Lambda)$ remains, nevertheless, detectable. In this case, the dissipative structure of the system is fully "damped" due to the negative definiteness of the matrix $S$. Then, there is no need for an output estimation error injection for complementing, or enhancing, the system's natural dissipative structure. The slave is designed as

$$
\left[\begin{array}{l}
\dot{\xi}_{1} \\
\dot{\xi}_{2} \\
\dot{\xi}_{3}
\end{array}\right]=\left[\begin{array}{ccc}
0 & \frac{1}{2} \sigma & 0 \\
-\frac{1}{2} \sigma & 0 & -x_{1} \\
0 & x_{1} & 0
\end{array}\right] \frac{\partial H}{\partial \xi}+\left[\begin{array}{ccc}
-\sigma^{2} & \frac{1}{2} \sigma & 0 \\
\frac{1}{2} \sigma & -1 & 0 \\
0 & 0 & -b
\end{array}\right] \frac{\partial H}{\partial \xi}+\left[\begin{array}{c}
0 \\
r x_{1} \\
0
\end{array}\right]
$$

and the synchronization error is therefore governed by the globally asymptotically stable system

$$
\left[\begin{array}{l}
\dot{e}_{1} \\
\dot{e}_{2} \\
\dot{e}_{3}
\end{array}\right]=\left[\begin{array}{ccc}
0 & \frac{1}{2} \sigma & 0 \\
-\frac{1}{2} \sigma & 0 & -x_{1} \\
0 & x_{1} & 0
\end{array}\right] \frac{\partial H}{\partial e}+\left[\begin{array}{ccc}
-\sigma^{2} & \frac{1}{2} \sigma & 0 \\
\frac{1}{2} \sigma & -1 & 0 \\
0 & 0 & -b
\end{array}\right] \frac{\partial H}{\partial e} \text {. }
$$

If the negative real part of the observable eigen values, related to the constant dissipation structure matrix of the error dynamics, must be enhanced, one can still use the above observer but now including an output reconstruction error injection term. The resulting observer (slave) is given by

$$
\left[\begin{array}{l}
\dot{\xi}_{1} \\
\dot{\xi}_{2} \\
\dot{\xi}_{3}
\end{array}\right]=\left[\begin{array}{ccc}
0 & \frac{1}{2} \sigma & 0 \\
-\frac{1}{2} \sigma & 0 & -y \\
0 & y & 0
\end{array}\right] \frac{\partial H}{\partial \xi}+\left[\begin{array}{ccc}
-\sigma^{2} & \frac{1}{2} \sigma & 0 \\
\frac{1}{2} \sigma & -1 & 0 \\
0 & 0 & -b
\end{array}\right] \frac{\partial H}{\partial \xi}+\left[\begin{array}{c}
0 \\
r y \\
0
\end{array}\right]+\left[\begin{array}{c}
K_{1} \\
K_{2} \\
K_{3}
\end{array}\right] e_{1}
$$

The asymptotically stable reconstruction error dynamics is then governed by

$$
\begin{aligned}
& {\left[\begin{array}{c}
\dot{e}_{1} \\
\dot{e}_{2} \\
\dot{e}_{3}
\end{array}\right]=\left[\begin{array}{ccc}
0 & \frac{1}{2} \sigma\left(1+K_{2}\right) & \frac{1}{2} \sigma K_{3} \\
-\frac{1}{2} \sigma\left(1+K_{2}\right) & \frac{1}{2} \sigma K_{3} & -y \\
-\frac{1}{2} \sigma K_{3} & y & 0
\end{array}\right] \frac{\partial H}{\partial e}} \\
& +\left[\begin{array}{ccc}
-\sigma\left(\sigma+K_{1}\right) & \frac{1}{2} \sigma\left(1-K_{2}\right) & -\frac{1}{2} \sigma K_{3} \\
\frac{1}{2} \sigma\left(1-K_{2}\right) & -1 & 0 \\
-\frac{1}{2} \sigma K_{3} & 0 & -b
\end{array}\right] \frac{\partial H}{\partial e} .
\end{aligned}
$$


One may now specify the values of $K_{i} j=1,2,3$ in order to guarantee a faster asymptotic stability to zero of the state reconstruction error trajectories.

\section{SYNCHRONIZATION STABILITY ANALYSIS}

In this section, we examine the stability of the synchronization error (11) between the Lorenz system (7) and observer (10). A necessary and sufficient condition for global asymptotic stability to zero of the estimation error is given by the following theorem.

Theorem 1 (Sira-Ramírez and Cruz-Hernández [14]) The state $x$ of the nonlinear system (7) can be globally, exponentially estimated, by the state $\xi$ of the observer (10) if and only if there exists a constant matrix K such that the symmetric matrix

$$
[W-K C]+[W-K C]^{T}=[S-K C]+[S-K C]^{T}=2\left[S-\frac{1}{2}\left(K C+C^{T} K^{T}\right)\right]
$$

is negative definite.

\section{PHYSICAL SYSTEM IMPLEMENTATION AND EXPERIMENTAL RESULTS}

In this section, we describe the physical implementation of the synchronization and communication schemes. Figure 2 shows the analog circuit implementation of the Lorenz equations ( 7 ).

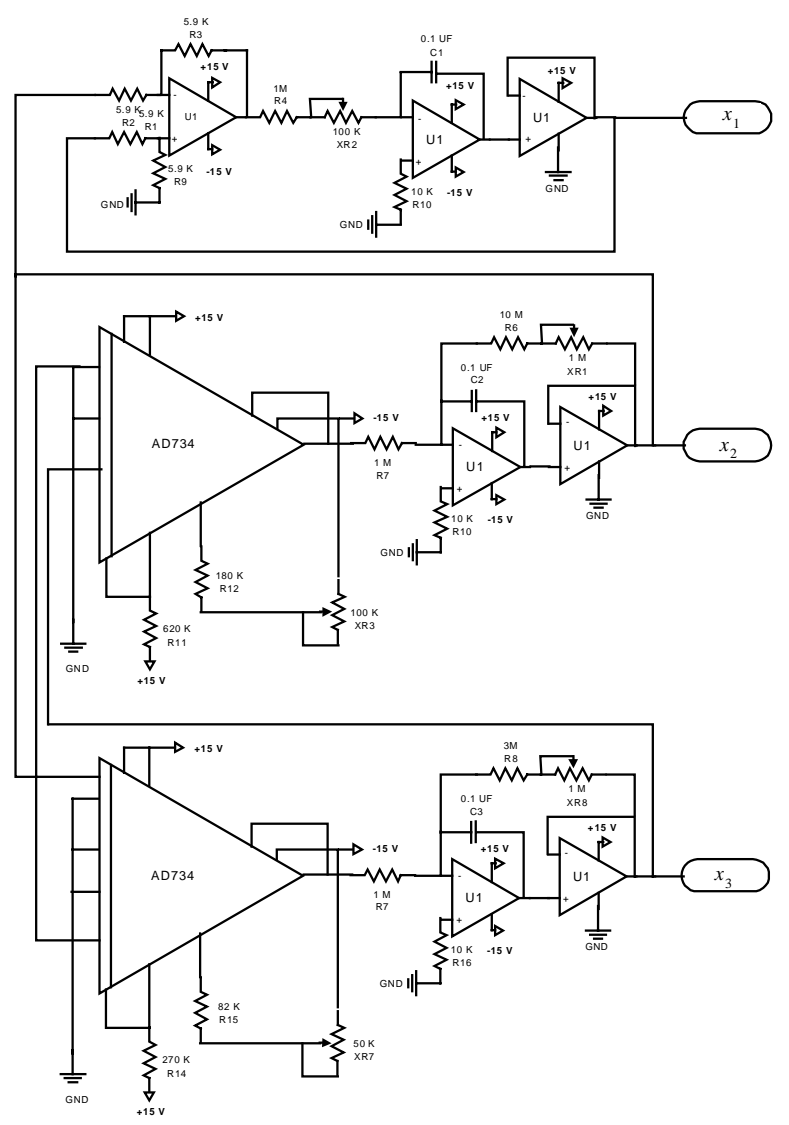

Figure 2. Analog circuit implementation of the Lorenz system 


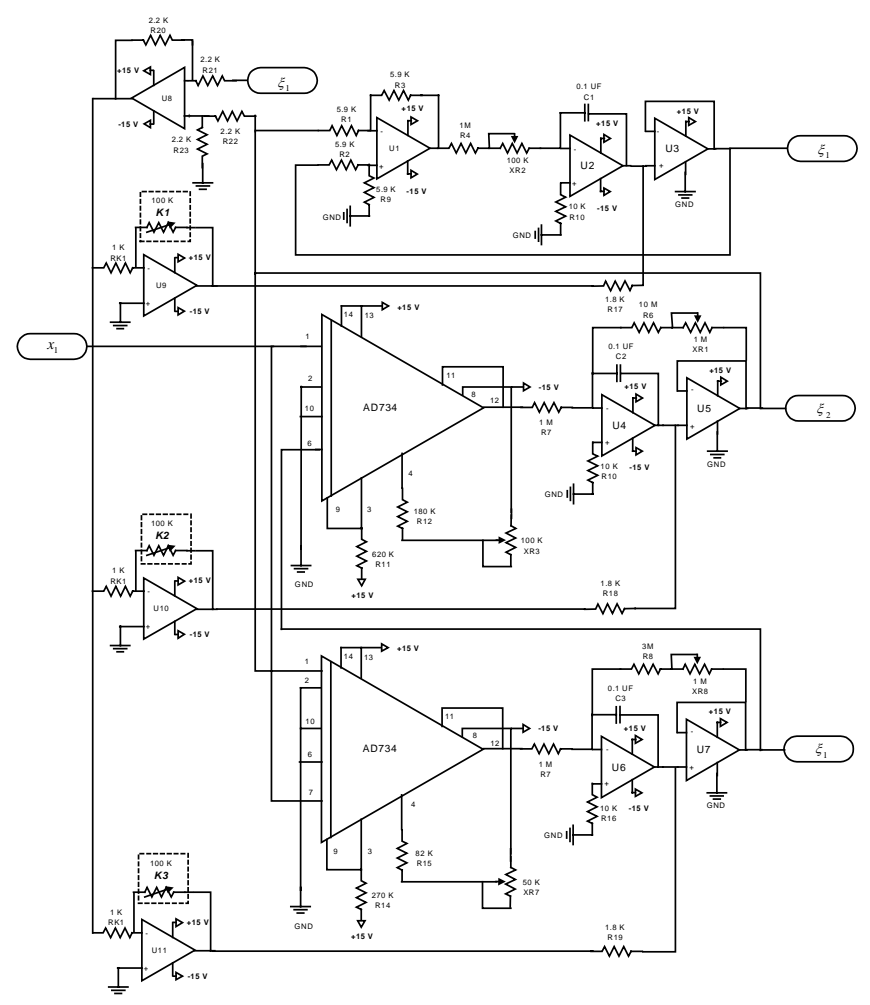

\section{Figure 3. Experimental set-up of Generalized Hamiltonian systems and observer approach to synchronize the Lorenz circuit}

\subsection{Synchronization}

The experimental set-up of Generalized Hamiltonian system approach and observer to synchronize the Lorenz circuits is shown in Figure 3. The basic idea of this scheme is as follows: a voltage $\pm 15 \mathrm{~V} d c$. Is injected into the Lorenz circuit (master system) which modifies the voltage $x_{i}(t)$. This voltage $x_{i}(t)$ is then used as driving signal on the Lorenz circuit (slave system) across I.C. AD734. The signal error $e_{l}(t)=x_{i}(t)-\xi_{1}(t)$, is injected into the block of U8. The choice of gains $K_{t}$ $=50$ and $K_{2}=K_{3}=0$ was used. Figure 4 shows the quality of synchronization between the master and slave circuits. The horizontal axis corresponds to $x_{t}(t)$ of the master and the vertical axis to $\xi_{1}(t)$ of the slave.

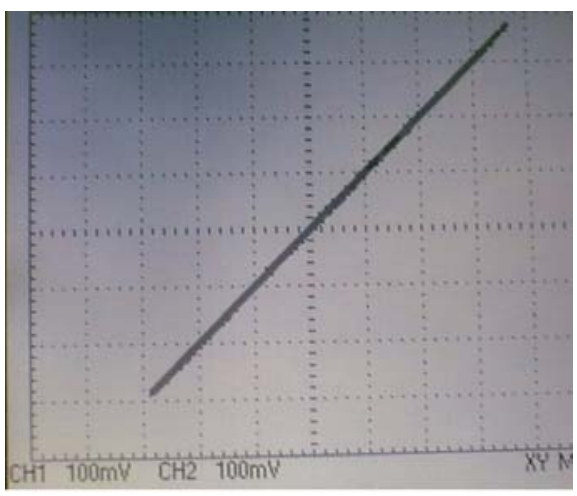

Figure 4. Synchronization between the master and slave circuits: $x_{1}(t)$ of the master (horizontal axis) versus $\xi_{1}(t)$ of the slave (vertical axis) 


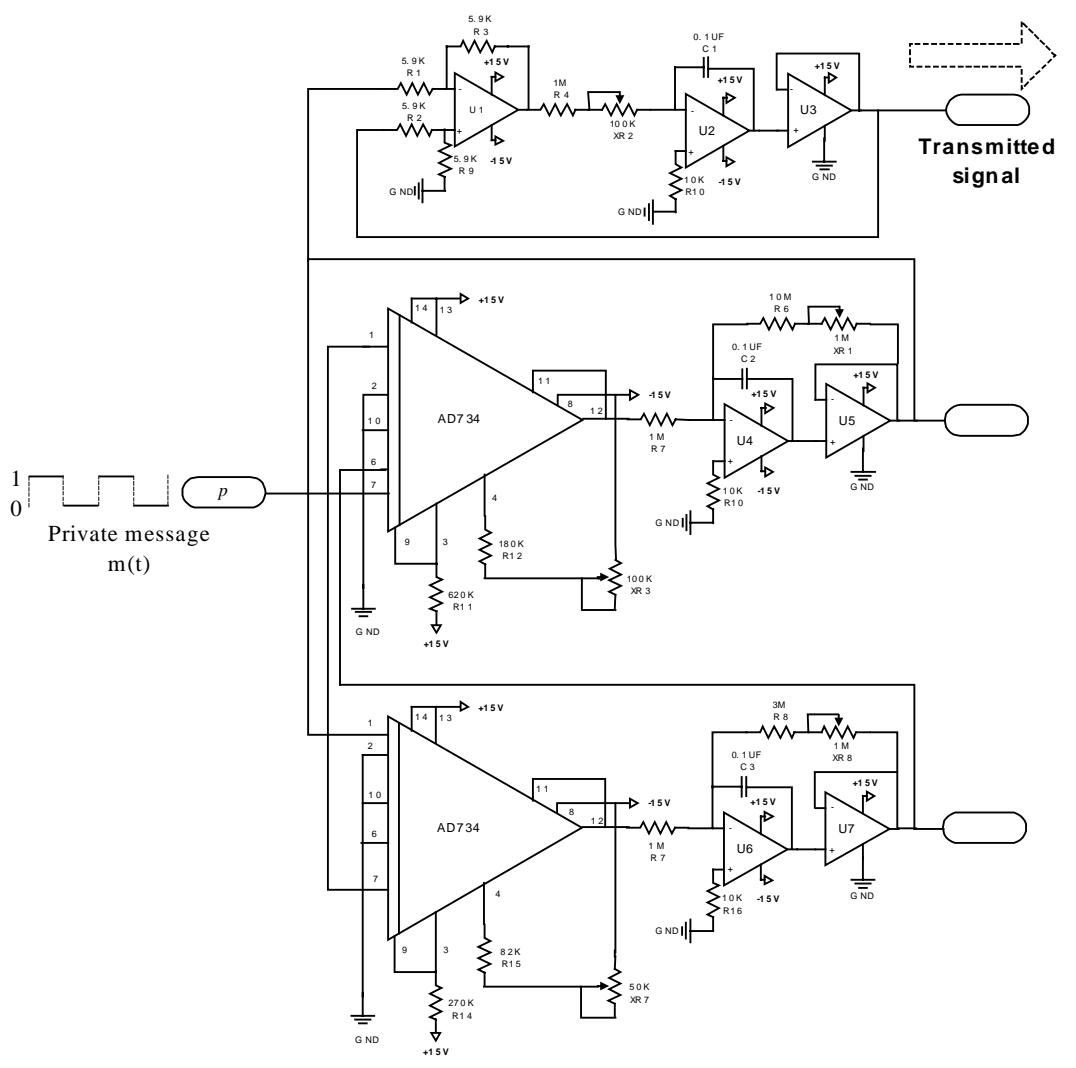

Figure 5 Experimental set-up to transmit binary signals by chaotic switching

\subsection{Binary communication}

The experimental set-up to transmit binary signals by chaotic switching is shown in Figure 5. The basic idea of this scheme is as follows: We generate two chaotic attractors of the Lorenz circuit (Eq. (7)), utilizing a square signal of $7 \mathrm{~V}$ dc. and $3 \vee \mathrm{dc}$. With these values, the Lorenz circuit exhibits two different but qualitatively similar chaotic attractors (to encoding "1" or "0" ). Figure 6 shows the transmission of an alternating sequence of "1" and "0" (transmitted signal $x_{i}(t)$. Figure 7, top of figure: shows binary signal to be transmitted. Bottom of figure shows the original information is recovered by synchronization error detection at the receiver.

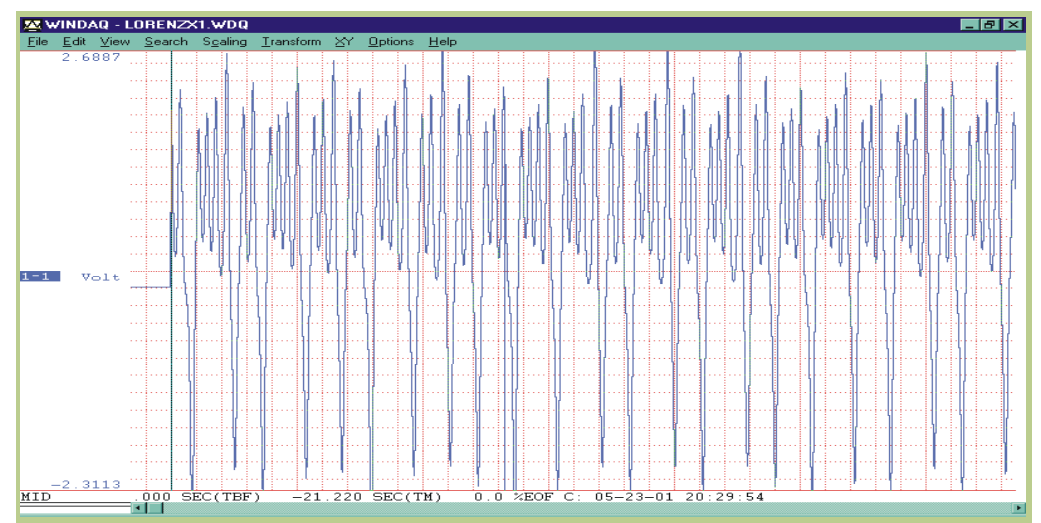

Figure 6. Transmitted signal $x_{1}(t)$ to the receiver 


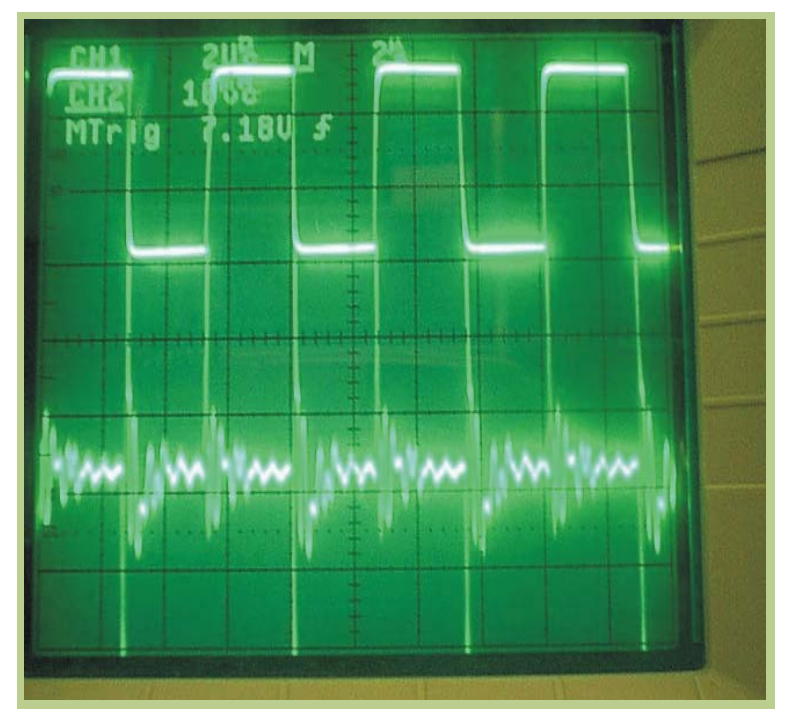

Figure 7. Top of figure: Binary signal to be transmitted. Botom of figure:Sinchronization error detection $e_{1}=x_{1}+\xi_{1}$

\section{CONCLUDING REMARKS}

In this paper, we have developed an experimental study of practical realization to transmit binary signals using synchronized chaos. Synchronization of two Lorenz circuits was obtained from the perspective of Generalized Hamiltonian systems and observer including dissipation and destabilizing terms provided in [14]. The approach allows to give a simple design procedure for the receiver system and clarifies the issue of deciding on the nature of the output signal to be transmitted. Experimental results shown that this approach is indeed suitable for synchronization of Lorenz circuit and can be easily applied to experimental private communication systems (e.g., by chaotic switching), see [23] and [24]. In addition, we have shown that the experimental results (on synchronization and communication) are in close accordance with the numerical simulations given in [22], where a similar approach was used to synchronize the Lorenz system to transmit private binary signals by chaotic switching.

In a forthcoming article we will be concerned with a physical implementation of the method combining with conventional cryptographic schemes to enhance the security of low-dimensional chaos-based secure communication systems.

\section{ACKNOWLEDGMENT}

This work was supported by the CONACYT, México, under Research Grant 31874-A.

\section{REFERENCES}

[1] Pecora L.M. \& Carroll,T.L. "Synchronization in chaotic systems", Phys. Rev. Lett. 64, 821-824, 1990.

[2] Chua,L.O., Kocarev, Lj., Eckert, K. \& Itoh, M. "Experimental chaos synchronization in Chua's circuit", Int. J. Bifurc. Chaos 2(3), 705-708, 1992.

[3] Kocarev, L., Halle, K.S., Eckert, K., Chua, L.O. \& Parlitz, U. "Experimental demostration of secure communications via chaotic synchronization," Int. J. Bifurc. Chaos 2(3), 709-713, 1992.

[4] Ogorzalek, M.J. "Taming chaos-Part I: Synchronization", IEEE Trans. Circuits Syst. I, 40(10), 693-699, 1993.

[5] Wu, C.W. \& Chua, L.O. "A simple way to synchronize chaotic systems with applications to secure communication systems", Int. J. Bifurc. Chaos 3(6), 1619-1627, 1993.

[6] Ding, M. \& Ott, E. "Enhancing synchronism of chaotic systems," Phys. Rev. E49(2), 945-948, 1994. 
[7] Feldmann, U. "Communication by chaotic signals: The inverse system approach," Int. J. Circuit Theory and Applications 24, 551-579, 1996.

[8] Nijmeijer, H. \& Mareels, I.M.Y. "An observer looks at synchronization", IEEE Trans. Circuits Syst. I, 44(10), 882890, 1997.

[9] Special Issue on "Chaos synchronization and control: Theory and applications", IEEE Trans. Circuits Syst./ 44 (10), 1997.

[10] Fradkov, A.L., Markov, A. \& Yu, A. P. "Adaptive synchronization of chaotic systems based on speed gradient method and passification", IEEE Trans. Circuits Syst. /44(10), 905-917, 1997.

[11] Cruz C. \& Nijmeijer, H. "Synchronization through extended Kalman filtering", New Trends in Nonlinear Observer Design, eds. Nijmeijer, H. \& Fossen, T.I., Lecture Notes in Control and Information Sciences 244 (Springer-Verlag), 469-490, 1999.

[12] Cruz C. \& Nijmeijer, H. "Synchronization through filtering", Int. J. Bifurc. Chaos 10(4), 763-775, 2000.

[13] Special Issue on "Control and synchronization of chaos", Int. J. Bifurc. Chaos 10(3-4), 2000.

[14] Sira-Ramírez H. \& Cruz, C. "Synchronization of chaotic systems: A generalized Hamiltonian systems approach", Int. J. Bifurc. Chaos 11(5), 1381-1395, 2001. And in Procs. Of American Control Conference, June 28-30, 2000, Chigago, III., USA, 769-773.

[15] Pikovsky, A. Rosenblum, M. \& Kurths, J. "Synchronization: A universal concept in nonlinear sciences", Cambridge University Press, 2001.

[16] Aguilar A. \& Cruz, C. "Synchronization of two hyperchaotic Rossler systems: Model-matching approach", WSEAS Transactions on Systems, 1(2), 198-203, 2002.

[17] Cuomo, K.M. Oppenheim, A.V. \& Strogratz, S.H. "Synchronization of Lorenz-based chaotic circuits with applications to communications", IEEE Trans. Circuits Syst. // 40(10), 626-633, 1993.

[18] Parlitz, U. Chua, L.O. Kocarev, Lj. Halle, K.S. \& Shang, A. "Transmission of digital signals by chaotic synchronization", Int. J. Bifurc. Chaos 2(4), 973-977, 1992.

[19] Dedieu, H., Kennedy, M. \& Hasler, M. "Chaotic shift keying: Modulation and demodulation of a chaotic carrier using self-synchronizing Chua's circuits," IEEE Trans. Circuits Syst. // 40(10), 634-642, 1993.

[20] Yang T. \& Chua, L.O. "Secure communication via chaotic parameter modulation", IEEE Trans. Circuits Syst. I 43(9), 817-819, 1996.

[21] Lorenz, E.N. "Deterministic nonperiodic flow," J. Atmospheric Sci. 20, 130-141, 1963.

[22] Posadas C. \& Cruz, C. "Transmisión de información privada sincronizando osciladores caóticos hamiltonianos", Congreso Nacional de la Asociación de México de Control Automático, A.C., May 3-4, 2001, San Luis Potosí, S.L.P., México.

[23] Posadas C. \& Cruz, C. "Transmisión de información privada sincronizando osciladores caóticos hamiltonianos", Congreso Nacional de la Asociación de México de Control Automático, A.C., May 3-4, 2001, San Luis Potosí, S.L.P., México,

[24] Posadas, C. Cruz, C. \& Núñez, R. "Experimental realization of binary signals transmission based on synchronized Lorenz circuits", ProcS. of the 2n WSEAS Int. Conf. on Measurement, Control, Circuits and Systems, May 12-16, 2002, Cancún México. 
Authors Biography

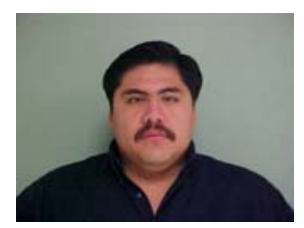

\section{Cornelio Posadas Castillo}

Was born in Poza Rica, Veracruz, México, in March 10 of 1973. He received the B. Sc. degree in Control and Computation from the Faculty of Mechanic and Electrical Engineering of the Autonomous University of Nuevo León, Monterrey, México, in 1997, and M. Sc. degree in Electronics and Telecommunications with mayor in Control, from Electronics and Telecommunications Department of Scientific Research and Advanced Studies of Ensenada, México, in 2001. Since 1997, he has been Associated Professor at the Autonomous University of Nuevo León, Monterrey, México.His research interests include control and synchronization of chaotic systems and private communications.

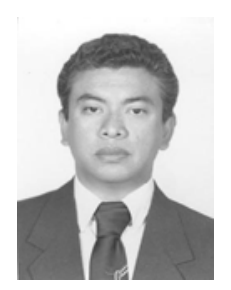

\section{César Cruz Hernández}

Received the M.Sc. and Ph.D. degrees in electrical engineering both from CINVESTAV, México, in 1991 and 1995, respectively. Since 1995, he is with the Department of Electronics and Telecommunications of the Scientific Research and Advanced Studies of Ensenada (CICESE), where he is currently Professor of Automatic Control. His research interests include multimode oscillations of coupled oscillators, nonlinear systems analysis, and synchronization and control of complex dynamical systems.

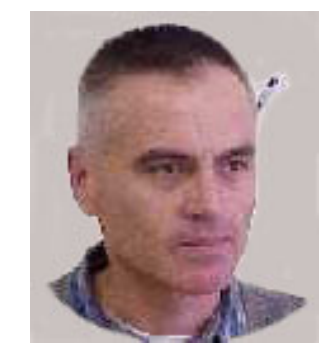

Ricardo F. Núñez Pérez

M.Sc. in Electronics and Instrumentation (87), from CICESE, México, and B.Sc. on Electronic Engineering (80), from UABC, México (Honors at both degrees). Since 1987 to date is a faculty member of the Electronics \& Telecommunications Department, CICESE Research Center. He was recipient of the First Prize at the National Contest on Design of Electronic Equipment Applied to Electric Sector by IIE, CONACYT, SEP and CFE $(88,90)$. Research Areas: Electronics Instrument Developing for Chaotic Circuits Studies and Applications, and Vibrations and Chaotics Signals DSP Applications. 